\title{
THE SURGICAL TREATMENT OF CONGENITAL HEART DISEASE
}

\author{
$B y$ O. S. Tubbs, F.R.C.S. \\ Brompton, and St. Bartholomew's Hospitals
}

The surgical treatment of congenital abnormalities of the heart and great vessels began I I years ago, when Gross (Gross and Hubbard, 1939), of Boston, U.S.A., successfully closed a patent ductus arteriosus by operation.

Six years later Crafoord (Crafoord and Nylin, 1945) in Stockholm succeeded in providing a normal circulation for a patient suffering from coarctation of the aorta by resecting the stenotic part of the aorta and performing an end-to-end anastomosis. During the same year Blalock (Blalock and Taussig, I945), of Baltimore, reported his now famous operation of anastomosis of the end of the divided subclavian artery to the side of the pulmonary artery, which gives immense relief to those cases with a deficient blood flow to the lungs and consequent cyanosis, particularly to those patients with Fallot's tetralogy.

Shortly afterwards Potts (Potts, Smith and Gibson, 1946), of Chicago, described another method of increasing the blood supply to the lungs, i.e. by side-to-side (lateral) anastomosis between the aorta and the pulmonary artery.

Some British surgeons (Sellors, I948; Brock, 1948) were not content with this indirect method of increasing the pulmonary blood flow and consequently attempted, with success, to relieve the pulmonary stenosis by section of the valve in those instances where the narrowing was limited to the valve. Subsequently Brock (1949) devised and practised a most ingenious method of enlarging the lumen of the pulmonary conus in those cases where the stenosis was proximal to the valve (' infundibular stenosis').

Yet other congenital lesions have been successfully treated by operation, e.g. auricular and ventricular septal defects (Murray, 1948), so that it is obvious that the scope of surgery in the treatment of congenital malformations of the heart and great vessels has by no means reached its limit. In addition, surgical techniques are constantly being improved, so that the operations are not only increased in safety; but the possibilities of surgery may be extended; thus Gross's (Gross, Bill and Peirce, 1949) report of the successful use of an aortic graft to fill the gap in cases of coarctation involving too great a length of the aorta to make end-to-end anastomosis possible may result in these previously inoperable cases becoming amenable to surgery.

Likewise Barrett's report (1949) of obtaining an increased blood supply to the lungs by causing the formation of vascular pleural adhesions may make it possible to help those children who have complete, or almost complete, absence of a pulmonary artery and in whom an anastomotic operation or direct operation to relieve the pulmonary stenosis is an impossibility.

But it is most important that we should retain our equilibrium amidst this whirlwind of progress lest we hasten too fast to the detriment of our patients. In assessing the results of operative treatment of the cyanotic cases we must remember the improvement in function which often follows encouragement, education in increasing exercise tolerance and physiotherapy. In selecting cases for operation the operative hazards must be contrasted with the utmost care against the natural risks of the disorder. And, perhaps most important of all, it is essential that the surgeon who elects to undertake this form of surgery should make an intensive study of the technical problems involved: such study should include practice on the cadaver and, wherever possible, on the living animal. It is inexcusable "to have a crack at it " without proper training.

\section{Patent Ductus Arteriosus}

The ductus which remains patent in postnatal life in the absence of other congenital vascular abnormalities endangers the life of the owner because of the risk of (I) chronic heart failure, (2) sudden death from acute heart failure or rupture of an aneurysm of the pulmonary artery and (3) subacute bacterial endarteritis.

Apart from these risks to life, there may be retarded growth and development due to a deficient systemic circulation, although, in my experience, this is not so frequent as the literature would suggest.

Penicillin and streptomycin have removed the sting from bacterial infection, as, with rare exceptions, the bacteria can be destroyed with a proper course of the appropriate drug and there is little 
danger of valvular damage if treatment is started early, for the vegetations are primarily situated in the pulmonary artery or the pulmonary end of the ductus. Likewise, embolism is not of serious consequence, as the emboli are carried into the lung and not the systemic circulation. The fact that about one-third of the patients with a patent ductus sooner or later become infected may not, therefore, be sufficient reason to advocate prophylactic surgical closure of an uncomplicated patent ductus.

However, the possibility of other catastrophes and the relatively high incidence of heart failure have convinced me and the cardiologists for whom I work that it is right to recommend operation in all cases of uncomplicatod patent ductus at about the age of seven. If there are signs of incipient heart failure, e.g. cardiac enlargement, at an earlier age, operation should not be postponed. This policy can only be justified if the operative mortality is exceedingly low, i.e. less than 3 per cent.

If a patient is first seen after the onset of infection, the infection should be eradicated by chemotherapy and the ductus closed subsequently to prevent recurrence. This is safer than operating forthwith, as operation undertaken while infection persists only cures about 60 per cent. of the cases (Tubbs, 1944). If the bacteria were resistant to all chemotherapeutic drugs (which must be of extreme rarity), it would undoubtedly be right to operate in the presence of the infection.

Patency of the ductus is usually discovered accidentally, e.g. at a school medical examination, as there is no cyanosis, no clubbing and often little or no limitation of exercise capacity.

The presence of a continuous ' machinery' or 'humming-top' murmur which is maximal in the second left intercostal space is recognized as the classical sign of a patent ductus. All the other described signs are merely corroborative evidence. A murmur confined to systole is almost certainly due to a lesion other than patency of the ductus.

Blood pressure readings are valuable in indicating the calibre of the ductus for the diastolic pressure and the bore of the ductus are in inverse proportion, i.e. a low diastolic figure indicates a ductus with a large lumen. As the heart is more likely to fail if the leak from the aorta to the pulmonary artery is large, the finding of a low diastolic pressure may influence one to operate earlier than would otherwise be the case.

The operation is performed through a left postero-lateral incision and the pleural cavity is entered through the fourth intercostal space. This approach gives better exposure than the old anterior incision and the scar is much less notice- able. After retracting the lung and incising the mediastinal pleura behind and parallel to the phrenic nerve, a slight fusiform dilatation of the aorta in the region of the ductal attachment will be seen. The connective tissue and aortic adventitia are picked up with two long haemostatic clamps just in front of the vagus and incised. By gentle backward retraction of the more posterior of the two clamps the connective tissue is raised a little from the aorta and this draws the vagus and recurrent laryngeal nerves away from the region of the ductus. Slight forward and lateral traction is applied to the other clamp and the connective tissue is dissected from the aorta, the ductus and a small part of the anterior wall of the pulmonary artery: by keeping close to the wall of the ductus, damage to the process of serous pericardium which usually overlaps the pulmonary end of the ductus is avoided. Now that the correct plane has been entered isolation of the rest of the ductus by blunt dissection is relatively easy. The best method of obliterating the ductus is not universally agreed. Simple ligation is certainly followed in rare instances by recanalization and Gross (1944) therefore routinely divides the ductus and sutures the two ends. For some time I have ligated the pulmonary and aortic ends with floss silk and subsequently used a transfixion ligature of thread at the mid-point of the ductus. None of the cases treated in this way has recanalized.

\section{Coarctation of the Aorta}

Two types of coarctation of the aorta have been described - infantile' and 'adult.' In the latter there is a narrow annular constriction of the aorta at the site of attachment of the ligamentum arteriosum, and the trunk and lower limbs receive most or all of their blood supply through collateral channels developed in the chest wall. It is this type which may be amenable to surgery. In the infantile type a longer segment of the aortic arch is narrowed and the distal part of the body receives most of its blood supply from the pulmonary artery through a persistently-patent ductus which opens into the aorta distal to the narrowing; other congenital abnormalities are frequently associated and death usually occurs during the first year of life.

It is fallacious to suppose that all cases of coarctation seen in adults or adolescence conform exactly to the classical description; the narrowing occasionally involves several centimetres of the aorta and may extend proximal to the origin of the left subclavian artery. Also, in about ro per cent. of cases, the ductus retains a lumen which more commonly communicates with the aorta proximal to the coarctation; the blood flow is, however, from the aorta to the pulmonary artery, i.e. in 
the opposite direction to that which occurs in the infantile type.

The well-known secondary effects of coarctation of the aorta are (I) a raised blood pressure in the brachio-cephalic circulation, (2) a diminished blood pressure in the lower limbs, (3) a strain on the left side of the heart and (4) the development of a collateral circulation in the chest wall.

Symptoms may result from the hypertension in the brachio-cephalic circulation, e.g. general weakness, headache, vertigo, palpitations and visual disturbance. The hypotension in the lower limbs may cause cold feet and a feeling of weakness of the legs; occasionally a mild form of intermittent claudication is experienced. Symptoms of cardiac failure may occur as a consequence of the strain on the left side of the heart; sometimes these symptoms come on very rapidly in a patient who has previously noticed very little limitation in his exercise tolerance, e.g. a boy of 19 who was admitted to. hospital in advanced congestive failure, from which he soon died, had been playing tennis without discomfort nine months previously.

Symptoms due to bacterial aortitis or endocarditis may also be superimposed on those of the uncomplicated coarctation.

However, many patients, especially young people, with aortic coarctation may complain of no symptoms at all.

On examination there is usually excessive pulsation in the neck and upper limbs. Tortuous pulsating vessels in the axillae and interscapular regions may be palpable or visible, but they are not always present, especially in children.

A systolic murmur is commonly heard over the precordium and often posteriorly as well. Crafoord (1947) believes that this results from the collateral vessels and not from the aortic constriction, but it is probable that either or both may be responsible. Pulsation of the abdominal aorta and of the femoral, popliteal, posterior tibial and dorsalis pedis arteries is much reduced or absent; if it is present in the femoral arteries, the pulse here will be appreciably later than that in the radial arteries. This reduced pulsation can be recorded by oscillometry.

The blood pressure in the arms is frequently elevated above $200 \mathrm{~mm}$. Hg. systolic, although much lower figures are common in children. Exercise may increase the systolic pressure by as much as $100 \mathrm{~mm}$. Hg. The importance of considering the possibility of coarctation in every case of 'essential hypertension' cannot be overemphasized, especially in young people.

A teleroentgenogram may show enlargement of the heart, but it will almost certainly show (I) a reduction or absence of the shadow due to the aortic arch and (2) a bulge along the upper part of the left border of the superior mediastinum due to dilatation of the left subclavian artery. Notching of the lower borders of some of the ribs may be seen due to pressure from the dilated and tortuous intercostal arteries, but this is by no means always present.

Angiocardiography may fail to show the site and length of the aortic constriction, as the radioopaque substance is often too much diluted by the time it reaches the aorta, but retrograde injection through a catheter introduced through the radial or axillary arteries may show the nature of the constriction and thus provide information as to the feasibility of resection and end-to-end anastomosis.

The most difficult problem is to decide which cases should be recommended for surgical treatment, for the operative mortality in the most expert hands is 10 to I 5 per cent. (Gross, 1949; Blalock, 1949; Crafoord, 1947). This has to be balanced against the risks of the abnormality if left untreated. Maud Abbott (1936) found that the average age at death of the adult type of coarctation was 32. Reifenstein et al. (Reifenstein, Levine and Gross, 1949) studied ro4 cases in which coarctation had been noted at autopsy and found that:

I. 26 per cent. lived a long and often active life,, some to advanced old age without symptoms.

2. 22 per cent. died from bacterial aortitis or endocarditis.

3. 23 per cent. died suddenly from aortic rupture.

4. 28 per cent. died as a result of the hypertensive state (cardiac failure was responsible about twice as frequently as intracranial haemorrhage).

The average age at death in the 104 cases was 35 ; the average age at death of those dying as a result of the coarctation was 30 .

It is therefore obvious that coarctation is a serious menace to life, but most cardiologists would probably recommend operation only to those cases with symptoms and those with evidence of extreme aortic narrowing.

It is undoubtedly unwise to operate under the age of 10, as the lumen at the site of anastomosis may not increase with age. On the other hand, the technical difficulties of operation increase after the age of 20 owing to degenerative changes in the wall of the aorta proximal to the stricture, so that, in spite of Blalock's (1949) report of a successful resection at the age of $4 \mathrm{I}$, it is preferable to operate during the teen-age.

At the operation excellent exposure can be obtained by opening the chest through the fifth rib bed, resecting the rib from the tip of the transverse process almost to the costal cartilage; in my experience it is unnecessary to divide other ribs or to ligate and divide any intercostal bundles. 
The mediastinal pleura is incised over the lower part of the left subclavian artery and over the aorta to about 2 in. below the constriction. The aorta is then mobilized above and below the stenosis,-taking the greatest care not to damage the greatly-dilated and thin-walled intercostal arteries. The ligamentum arteriosum which is frequently thicker than normal should be divided as soon as possible, as it usually draws the site of coarctation medially, so that its division greatly facilitates subsequent mobilization of the aorta. Often there is a dilated bronchial artery arising from the anterior wall of the aorta and this must be divided between ligatures. As the upper intercostal arteries run in a cephalad (and lateral) direction, they do not impede mobilization of the aorta distal to the coarctation in an upward direction, so that it is unnecessary to divide any of them unless they arise from the part of the aorta to be excised or sufficiently close to the site of section as to interfere with the suturing.

After mobilization the aorta is clamped above and below the stricture, which is then excised; as the aorta distal to the stricture is frequently of greater calibre than it is above the narrowing, the site of section must be carefully adjusted in order to make the two openings approximately equal. An everting mattress suture through all coats of the aorta is used by most surgeons for joining the two aortic ends; this suture is continuous apart from two or three interruptions which are needed to avoid a purse-string effect.

If the aortic stricture is very close to the origin of the left subclavian artery, it may be necessary to include the base of this vessel in the proximal aortic clamp; alternatively, two separate clamps may be used-one across the aorta proximal to the origin of the left subclavian artery and the other across the subclavian artery itself.

If the stricture involves too great a length of the aorta to make end-to-end anastomosis possible, the dilated left subclavian artery may be divided as high up as possible and the proximal end anastomosed to the aorta distal to the stricture (Blalock and Park, 1944; Clagett, 1947). This operation obviously gives a less perfect result and is probably more hazardous than end-to-end anastomosis of the aorta, but a satisfacto $y$ fall in the brachio-cephalic pressure may follow, thus giving considerable relief to otherwise inoperable cases. If Gross's experimental work on the use of aortic grafts proves satisfactory in the humanand he has already reported six apparently successful results (Gross, 1949)-the need for this subclavio-aortic anastomosis will disappear.

The appearance of normal - even exaggeratedpulsation in the lower limbs is immediate following removal of the clamps, and the blood pressure in the arms falls considerably at the same time, but it may not reach normal limits for several weeks.

\section{Cyanotic Congenital Heart Disease}

The differential diagnosis of the various forms of congenital heart disease causing cyanosis is discussed in another article in this number, so that it is only necessary to consider which cases may benefit from operation and which investigations are needed from a purely surgical point of view.

Those patients with Fallot's tetralogy suffer from cyanosis partly as a result of the interventricular septal defect and overriding of the aorta and partly due to a deficient pulmonary blood flow consequent to the pulmonary stenosis (valvular or infundibular or both). Likewise, in tricuspid stenosis there are two factors in the cyanosis: (I) a right to left shift in the auricles and (2) a deficient blood flow to the lungs due to the failure in development of the right ventricle and pulmonary artery; in most cases the blood flow to the lungs depends on a patent ductus and collateral vessels or collateral vessels alone.

The anastomosis of a systemic vessel to the pulmonary artery would obviously increase the pulmonary blood flow in Fallot's tetralogy and, in the absence of a large ductus, in cases of tricuspid stenosis. If such an anastomosis is decided upon the relative merits of Blalock's subclavio-? pulmonary anastomosis and Potts's aorto-pulmonary anastomosis have to be considered. The technical difficulties of Blalock's operation under the age of three are so great that, if it is deemed imperative to operate under this age because of the severity of the lesion, most surgeons would elect to perform Potts's operation. In patients more than three years old there is no general agreement as to which operation gives the best results. On general principles it would seem a safer procedure to use the subclavian artery rather than the aorta for anastomosis; in addition, the risk of providing too large a communication between the systemic and pulmonary circulations is small if the anastomosis is made with the subclavian artery, whereas this risk is very real using the aorta unless the size of the opening is invariably within the limits (an aortic incision between $\frac{1}{4}$ in. and $\frac{5}{16}$ in., according to age) recommended by Potts (1948).

If the subclavian artery is to be used, Blalock strongly advises taking the one arising from the innominate artery in preference to that arising directly from the aorta, i.e. the operation is done on the right if there is a left-sided aortic arch, and on the left if the aortic arch is on the right (the aorta is right-sided in about one-third of the patients with Fallot's tetralogy). 
The reason for this recommendation is that, if the subclavian artery arising directly from the aorta is used for anastomosis, the vessel tends to form an acute angle at its origin, which interferes with the blood flow; this does not happen with the subclavian artery arising from the innominate artery. On the other hand, the subclavian artery arising directly from the aorta is longer and the divided end can be united more easily to the pulmonary artery without tension; for this reason there is a tendency amongst British surgeons to use this vessel, justifying the procedure on the grounds that the kink at the aortic origin is soon rectified after the lung is re-expanded. An additional advantage of doing the operation on the same side as the aortic arch is that a Potts's aortic anastomosis can be done if it is apparent that it would be impossible or difficult to use the subclavian artery.

It is therefore clear that pre-operative radioscopy with barium in the oesophagus is necessary to demonstrate the side of the aortic arch. It is also most helpful to have angiocardiograms which show the primury branches of the aortic arch, as it may then be possible to decide which systemic vessel would be most suitable for anastomosis.

The technique of anastomosis so beautifully performed and described by Blalock (1947) remains the standard, but some British surgeons have preferred to use an approach through a postero-lateral incision in the fourth intercostal space, as this gives better exposure and permits the performance of an aorta-pulmonary anastomosis should this appear to be more practicable, an alternative which is impossible through an anterior incision. But now that valvulotomy or infundibular resection (see later) are other possible alternatives it could be argued that these are only possible through an anterior approach. Blalock advises closure of the wound without pleural drainage, but I have always used closed drainage on my patients; this has the advantage of making post-operative aspiration unnecessary and I have not seen any harm result.

Valvulotomy (Brock, 1949) is carried out through an anterior incision through the third intercostal space. A large window in the pericardial sac is made and the infundibulum is incised between mattress sutures so as to allow the passage of a special valvulotome which is used to cut the stenosed valve. Bleeding from the incision in the infundibulum is controlled readily with the finger while sutures are placed to close the incision; the closure is reinforced by fixing the flap of parietal pericardium to the heart with the same sutures as were used to close the incision in the heart.

In those cases of infundibular stenosis where there is an interval of $\frac{1}{2}$ in. or more between the stenosis and the pulmonary valve, Brock (1949) has used a somewhat similar technique to enlarge the narrowing, except that a specially designed punch which actually cuts out part of the endocardium and heart muscle is used instead of a valvulotome.

The most indirect method of improving the blood flow to the lungs is that described by Barrett (1949). In those cases where he has found it impossible to perform an anastomotic operation he has removed the parietal pleura from the upper part of the chest and insufflated asbestos powder over the lung in order to promote the subsequent formation of vascular adhesions in which blood may be carried from the chest wall to the lung. The results reported were certainly encouraging, but it is difficult to believe that this procedure will ever replace more direct methods; however, it is a comfort to know that it is not necessarily impossible to provide some relief to those patients in whom operation reveals complete, or almost complete, absence of a pulmonary artery.

As there are now several alternative methods of improving the pulmonary blood supply in cases of Fallot's tetralogy it is necessary to decide, not only which patients are likely to benefit from operation, but also which operation is likely to give the greatest relief with the least risk.

Regarding the selection of patients for some form of operation, it is not possible to lay down clear-cut indications. If the lesion is not causing much disability and there is fair exercise tolerance, it is inadvisable to recommend operation. It is also the experience of all surgeons working on these cases that the risks of operation are infinitely greater in the older age group, so that patients over the age of 16 should not be accepted for surgery unless their incapacity is severe.

It is possible that valvulotomy will prove to give the best results in cases of pure valvular stenosis, that infundibular resection will be the correct procedure in cases where the stenosis is limited to the pulmonary conus and is not too close to the valve, and that some form of systemic-pulmonary anastomosis will be employed for patients in whom the stenosis cannot be relieved by direct operation, reserving Barrett's pleurectomy for those cases in which all other operations are impossible, but it is premature to forecast the future of these operations.

If the operation succeeds in providing a considerable increase in the pulmonary blood, the improvement in the patient's capacity is remarkably gratifying. The colour improves, the clubbing decreases, the classical 'squatting' ceases and the patient soon finds he has a great increase in exercise tolerance. Children who have been kept at home and have been a constant source of anxiety 
to their parents are often able to go to school and take part in the less strenuous games.

It is difficult to lay down criteria on which the value of the operative procedure itself may be judged, for, as already mentioned, the improvement in exercise capacity which may result from encouragement, physiotherapy and training-all of which precede and follow the operation-may be much greater than expected. Arterial oxygenation is not of great value, as this varies so much from time to time and it is particularly fallacious in children in whom the withdrawal of arterial blood samples may be accompanied by considerable physical exertion. The haemoglobin content of the blood and the volume of packed cells seem to me two of the most reliable scientific criteria on which the effect of the operation may be judged and, for this reason, these tests should always be included in the pre-operative investigation.

Finally, it is necessary to consider the risks of operation, but, in doing so, it is right that we should remember that the cardiac abnormalities themselves are most dangerous to the owners. When Blalock (Blalock and Bahnson, 1948) reported the results of his first 610 consecutive operations on patients with cyanotic congenital heart disease there was an over-all mortality of 17.7 per cent., but this series included all his pioneer work and also many very bad risk cases. In 433 cases in which an end-to-side subclaviopulmonary anastomosis was performed the mortality was 10.4 per cent.

At the present time a reasonable estimate of the operative risk would be to per cent.

\section{Septal Defects}

Septal defects occurring as the sole anomaly are said to have a relatively good prognosis, but Murray (1948) found evidence that this was not true about large interatrial and interventricular communications and undertook the remarkably courageous work of discovering some means of obliterating, or at least reducing, the hole between the right and left side of the heart. After much preparative experimental work on animals, he evolved techniques which were applied to human patients with very considerable success. In cases of interatrial defects multiple interrupted mattress sutures of silk are passed right through the heart in an antero-posterior direction in the line of the septum, so that when these are drawn up and tied linear portions of the anterior and posterior walls of the atria are approximated and the communication between the right and left side is obliterated. With interventricular defects a wide strand of fascia lata, together with a portion of tensor muscle, is passed through the heart in the position of the upper part of the interventricular septum (the site of the defect) and this graft is anchored to the front and back of the heart. In spite of this astonishing assault on the heart, Murray was able to report 17 cases submitted to operation with only one death when he delivered his Moynihan lecture at the Royal College of Surgeons of England in July 1949 .

Intensive research is being carried on with a view to providing relief to patients with other congenital cardiac abnormalities and further surgical advance is completely unpredictable. An attempt has been made to survey the progress so far achieved.

\section{BIBLIOGRAPHY}

ABBOTT, MAUD E. (1936), Atlas of Congenital Heart Disease, New York, American Heart Association.

BARRETT, N. R. (1949), Brit. Med. F., r, 699.

BLALOCK, A., and PARK, E. A. (r944), Ann. Surg., rr9, 445.

BLALOCK, A., and TAUSSIG, H. B. (1945), F. Am. Med. Ass., I28, 189 .

BLALOCK, A. (1947), F. Thor. Surg., 16, 244.

BLALOCK, A., and BAHNSON, H. T. (1948), Ann. of R. Coll. Surg. Eng., 3, 57.

BLALOCK, A. (r 949), in discussion of Gross's paper (1949), F. Am. Med. Ass., 139, 286.

BROCK, R. C. (1948), Brit. Med. F., I, I 12 I.

BROCK, R. C. (r949), Ibid., 2, 399.

CLAGETT, O. T. (1947), Proc. Staff Meet. Mayo Clinic, 22, 131. CRAFOORD, C., and NYLIN, G. (1945), $\mathcal{F}$. Thor. Surg., 14, 347. CRAFOORD, C., EJRUP, B., and GLADNIKOFF, H. (1947), Thorax, 2, 121 .

Gross, R. E., and HUBbARD, J. P. (r939), f. Am. Med. Ass., I12, 729.

GROSS, R. E. (1944), Surg. Gyn. Obst., 78, 36.

GROSS, R. E. (1949), भ. Am. Med. Ass., 139, 286.

GROSS, R. E., BILL, A. H. (Jr.), and PEIRCE, E. C. (I I) (r949), Surg. Gyn. Obst., 88, 6.

MURRAY, G. (1948), Ann. Surg., 128, 843.

POTTS, W. J., SMITH, S., and GIBSON, S. (1946), f. Am. Med. Ass., 132, 627.

POTTS, W. J. (1948), Ұ. Thor. Surg., r7, 223.

REIFENSTEIN, G. A., LEVINE, S. A., and GROSS, R. E. (1947), Am. Heart $\mathcal{F}$., 33, 146.

SELLORS, T. H. (1948), Lancet, r, 988.

TUBBS, O. S. (1944), Brit. F. Surg., 32, I. 